University of Wollongong

Research Online

Faculty of Informatics - Papers (Archive)

Faculty of Engineering and Information

Sciences

October 2003

\title{
Line segment distribution of sketches for Persian signature recognition
}

A. Chalechale

University of Wollongong

Alfred Mertins

University of Oldenburg, Germany, mertins@uow.edu.au

Follow this and additional works at: https://ro.uow.edu.au/infopapers

Part of the Physical Sciences and Mathematics Commons

\section{Recommended Citation}

Chalechale, A. and Mertins, Alfred: Line segment distribution of sketches for Persian signature recognition 2003.

https://ro.uow.edu.au/infopapers/56

Research Online is the open access institutional repository for the University of Wollongong. For further information contact the UOW Library: research-pubs@uow.edu.au 


\title{
Line segment distribution of sketches for Persian signature recognition
}

\author{
Abstract \\ A novel fast method for line segment extraction based on chain code representation of thinned sketches \\ (or edge maps) is presented and exploited for Persian signature recognition. The method has a parallel \\ nature and can be employed on parallel machines. It breaks the macro chains into several micro chains \\ after applying shifting, smoothing and differentiating. The micro chains are then approximated by straight \\ line segments. Length and position distributions of the extracted line segments are used to make a \\ compact feature vector for Iranian cursive signature. The feature vector is invariant under affine \\ transforms and can be used effectively in paperless office projects. Experimental results show fast \\ response and accurate recognition/retrieval rate.

\section{Disciplines} \\ Physical Sciences and Mathematics

\section{Publication Details} \\ This article was originally published as: Chalechale, A \& Mertins, A, Line segment distribution of sketches \\ for Persian signature recognition", Conference on Convergent Technologies for Asia-Pacific Region \\ (TENCON 2003), 15-17 October 2003, 1, 11-15. Copyright IEEE 2003.
}




\title{
Line Segment Distribution of Sketches for Persian Signature Recognition
}

\author{
Abdolah Chalechale ${ }^{1}$ and Alfred Mertins ${ }^{2}$ \\ ${ }^{1}$ School of Electrical, Computer and Telecommunications Engineering \\ University of Wollongong, Wollongong, NSW 2522 Australia \\ ac82@uow.edu.au \\ ${ }^{2}$ School of Mathematics and Natural Sciences \\ University of Oldenburg, Oldenburg, 26111 Germany \\ alfred.mertins@uni-oldenburg.de
}

\begin{abstract}
A novel fast method for line segment extraction based on chain code representation of thinned sketches (or edge maps) is presented and exploited for Persian signature recognition. The method has a parallel nature and can be employed on parallel machines. It breaks the macro chains into several micro chains after applying shifting, smoothing and differentiating. The micro chains are then approximated by straight line segments. Length and position distributions of the extracted line segments are used to make a compact feature vector for Iranian cursive signature. The feature vector is invariant under affine transforms and can be used effectively in paperless office projects. Experimental results show fast response and accurate recognition/retrieval rate.
\end{abstract}

\section{INTRODUCTION}

There are several low-level image primitives used in computer vision and scene analysis. One of the most useful primitives is elementary line segments (ELSs). A line segment as a primitive geometric object is frequently used in higherlevel processes such as object recognition, stereo matching, target tracking, image registration and also face recognition [1]. Different characteristics of line segments distribution can serve as useful features in image retrieval applications as well

An edge map is generally created in the early states in most image understanding systems. Although the edge points could be connected on the basis of image connectivity, proximity and orientation, such a representation has limited use since it lacks the compact analytic description of image features required for any vision tasks. The desired description must also overcome the problems of noise and scatter in the position of edge pixels [2].

Employing lineal features in an image instead of using edge points solely is noted widely in the literature [1], [2], [3], [4], [5], [6], [7]. Hough transform is formerly used for line detection. The main concept in this approach is based on voting, where local edges vote for all possible lines they are consistent with. The main advantage of this approach is that it enables us to detect collinear edge points even though they are all isolated. The method is useful in noisy images when local information around edge pixels is unreliable or unavailable. Another advantage of this method is that it has a global nature because a voting score assigned to each detected line is tallied up by considering all the edge pixels lying on the line. However, there are several problems with this approach. It has a relatively expensive cost based on memory usage and computation time. It also arises the so-called connectivity problem, where illusionary lines composed of accidentally collinear edge pixels are falsely detected [3], [4].

Burns et al. [5] introduced an approach for the extraction of straight lines in intensity images. It begins at the level of lines directly without going through the intermediate stage of first detecting local edges. It utilizes the gradient direction to partition the image into a set of support regions.

Nelson [6] proposed a recursive growing algorithm to the edges to identify the line segments in the images. Assfalg et al. [7] applied this method for semantic annotation of sports videos. In an another approach suggested by Etemadi [2], chains of edge points are obtained using the Marr-Hildreth edge finder. The chains are then segmented into pieces that are symmetric about their centroid and then combined to longer segments. A face feature representation, called Line Edge Map (LEM), is proposed in [1] to integrate the structural information with spatial information of a face image by grouping pixels of face edge maps to line segments.

In addition, there are several studies dealing with Farsi/Arabic character numeral recognition. Mowlaei et al. [8] used wavelet transform for feature extraction of Farsi/Arabic handwritten. In [9], a multilayered feed forward neural network is employed for segmentation and recognition. Fuzzy vector quantization [10], hidden Markov models [11], and statistical approaches [12] are used for Persian cursive script recognition.

Normalized central moments and skew and rotation invariant functions based on them have been used as powerful tools for shape description (see [13]). Dehghan and Faez [14] have evaluated the effectiveness of various moments in recognition of handwritten Persian characters. 


\section{TENCON $2003 / 12$}

Chain code histogram is introduced in [15] and utilized for shape recognition of irregular objects. It has been showed that similar objects are grouped together using the approach.

English signature analysis, verification and recognition have been studied extensively. They could be divided into two broad areas: on-line and off-line. Recently, a comparison between wavelet-based and function-based on-line signature verification has been reported by Da Silva and De Freitas [16] whilst Justino et al. [17] have focused on off-line signature classification using hidden Markov models. On the other hand, Persian signatures have a different essence from English ones. The former are cursive sketches usually independent of the person's name while the latter are often reshaped handwritten names.

This paper proposes a new fast method for line segment extraction and applies it to Persian cursive signature recognition. The input image is a thinned sketch or an edge map. The method has a parallel nature and can be used on parallel machines easily. It uses the chain codes in the input image, namely macro chains, for line segment detection. It then breaks the macro chains into several micro chains by employing the extreme points of the first derivative of shiftedsmoothed chain code function. The micro chains are then approximated by straight line segments. Length and position distributions of the line segments are combined to make a compact 80 -entry feature vector. The feature vector is used for retrieving Persian signatures in a hypothetical paperless office. It has been compared with two other methods and showed an accuracy rate of more than $97 \%$.

The rest of the paper is organized as follows. In the next section the proposed line segment and feature extraction method is described in detail. Section 3 exhibits the experimental results. Conclusions and further works are finally given in Section 4.

\section{CHAIN-BASED LINE SEGMENT EXTRACTION (CBLSE)}

In this section the details of the Chain-Based Line Segment Extraction (CBLSE) method are discussed. The input of the method is an edge map $I$ derived by Canny operator [18] on any real image. In addition, any thinned sketched image can also used as the input $I$. First, the start point of an 8 connectivity chain code (see [19]) is determined. The macro chain $A_{i}=\left\{a_{1}, a_{2} \ldots a_{n}\right\}, i=1,2, \ldots, m$, where $m$ is the - number of chains in $I$ and $n$ is the chain length, is obtained and put in a chain set $\left\{\dot{A_{i}}\right\}$. For each $A_{i}$ in $\{A\}$ we then apply the following steps (see Figure 1):

1. Eliminating Chain Noise: noisy points which make the chain over oscillating is eliminated by median filtering (such as first peak in Figure 1-b). Applying an order 3 onedimensional median filter on the vector $A_{i}$ reduces the effect of such points adequately.

2. Shifting Operation: the standard chain code representa- tion has the wraparound drawback. For example, a line along $-22.5^{\circ}$ direction is coded as $\{707070 \ldots\}$ and not via the more natural code $\{787878 \ldots\}$. To eliminate or reduce such wraparound, we produce a new modified code $B_{i}=\left\{b_{1}, b_{2} \ldots b_{n}\right\}$ for each $A_{i}=\left\{a_{1}, a_{2} \ldots a_{n}\right\}$ by a shifting operation [20] defined recursively as:

$$
\left\{\begin{aligned}
b_{1}= & a_{1} \\
b_{i}= & q_{i} \text { where } q_{i} \text { is an integer such that } \\
& \left(q_{i}-a_{i}\right) \bmod 8=0 \text { and } \\
& \left|q_{i}-b_{i-1}\right| \text { is minimized for } i=2,3, \ldots n .
\end{aligned}\right.
$$

The line along $-22.5^{\circ}$ direction is now coded as $\{787878 \ldots\}$. The comparison of Figure $1-b$ and $1-c$ shows the wraparound effect.

3. Smoothing Operation: the shifted chain code $B_{i}$ is then smoothed by a five-point Gaussian filter $\{0.1,0.2,0.4,0.2,0.1\}$ [20]. $\Gamma(\theta)$ is the resulted shifted and smoothed waveform where $\theta$ is the traversing variable (Figure 1-d).

4. First Derivative and Break Extraction: $d \Gamma / d \theta$ determines the rate of change of $\Gamma(\theta)$ with respect to $\theta$. The extreme points of this derivative are considered as break points $\left(\zeta_{i}\right)$, if they are grater than a threshold. Because the shiftedsmoothed function $d \Gamma / d \theta$ mostly has adjacent values with the difference of 0 or 1 , the maximum of Gaussian filter $(0.4)$ is depicted for the threshold (Figure 1-e).

The line segment $l_{i}$ which connects $\zeta_{i}$ to $\zeta_{i+1}$ is considered as the lineal approximation of the micro chain lays between the two points (Figure 1-f).

Although a polygonal approximation for the micro chain from $\zeta_{i}$ to $\zeta_{i+1}$ can be employed to obtain more line segments, experiments on many test data, carried out by the authors of this paper, showed that there is no significant improvement in the number of line segments by applying such polygonal approximation. In other words, there would be neither an objective nor a subjective significant difference in the final results by embedding such approximations/computations. Therefore the set $\left\{l_{i}\right\}$, where $l_{i}$ is the straight line segment connecting $\zeta_{i}$ to $\zeta_{i+1}$ will be used as the line segments set of chain $A_{i}$. The total collection of $l_{i}$ 's, say $L$, is the line segment set of the image/sketch $I$.

The lengths of the line segments in $L$ are rotation and translation invariant. Normalizing the length by maximum segment's length makes it scale invariant as well. In addition, the distance of each line segment's midpoint from the center of mass of the image is also rotation and translation invariant and the normalized one is scale invariant too.

For the purpose of Persian signature recognition, we employ these two principles to extract a discriminating and affine transforms invariant histogram. The histogram is made as follows.

1. For each segment in $L$, compute the length and the dis- 


\section{Image Processing / 13}

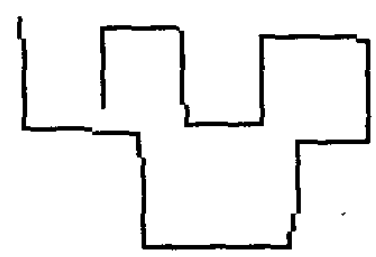

(a)

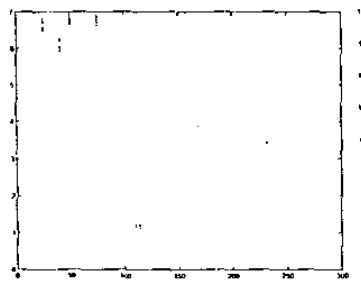

(b)

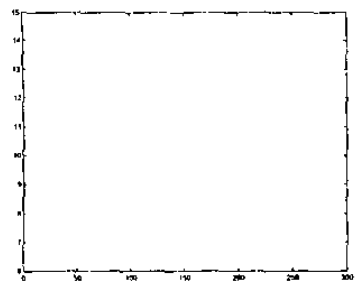

(d)

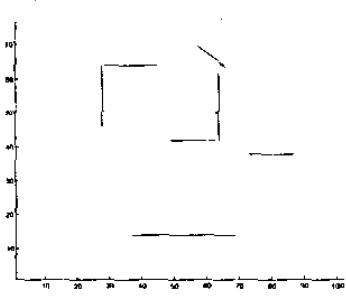

(f) (c)

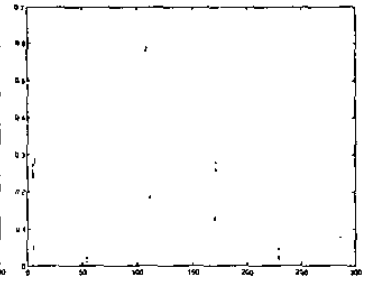

(e)
Figure 1. (a) an example contour, (b) chain code, (c) shifted chain code, (d) smoothed representation, (e) derivative and (f) thirteen extracted line segments

tance to the center of mass

2. Normalize the length with maximum length and the distance with size of bounding box.

3. Uniformly quantize the normalized length to 20 equal parts and the normalized distance to 4 equal parts.

4. Construct an 80-bin histogram $H$ and count the number of line segments in each bin according to its normalized length and the normalized distance.

For example, a line segment with normalized length equal to 0.83 and the normalized distance equal to 0.55 will be considered as a member of bin 57 .

\section{EXPERIMENTAL RESULTS}

We assumed an office with 50 employees and collected 50 Iranian cursive signatures (set $P$ ). Each signature in $P$ was scanned with $300 \mathrm{dpi}$ and then morphologically thinned. The histogram $H$ was obtained off-line for each signature using the proposes method (Section 2). Next, we collected signatures from the same persons at another time (set $Q$ ) and the histograms were obtained with the same stages. To evaluate the accuracy of the proposed method, we applied all signatures in $Q$ as queries while regarding $P$ as signature models.

As an alternative method, we constructed a 64-bin chain code histogram which is an extension of the 8-bin chain code histogram proposed in [15]. Here we look at two-digit chain code sequences and count the number of similar blocks in the signature chain code. For example, the number of occurrences of the " 00 " bitstream in the code was counted in bin 1. Similarly, the frequency of the " 27 " bitstream was stored in bin 24 and the frequency of the " 77 " bitstream in bin 64. Moreover, invariant moments [21], [14] that are used in a number of applications as powerful recognition tools are considered as another signature comparison criterion. The signatures are represented in terms of seven invariant function of moments $\left(\phi_{1}-\phi_{7}\right)$. The first six functions $\left(\phi_{1}-\phi_{6}\right)$ are invariant under rotation and the last one $\phi_{7}$ is both skew and rotation invariant [13].

We used the evaluation method introduced in [22] with Manhattan-Cityblock $\left(\ell_{1}\right)$ distance to obtain a quantitative value for the recognition rate. The method is based on average scoring and gives the score 1 to exact findings (rank 1) and the score 0 when the signature we are looking for has the worst rank ( 50 in this case). Any other ranks have a score between 1 and 0 , according to the position of the retrieved signature. Figure 2 shows some signatures in our test data. Table 1 exhibits the average scores of retrieving 50 -signatures, the time consumed to build histograms at the off-line stage and the time to produce the ranked list for an arbitrary signature during the on-line stage. As it can be seen, the proposed line segment distribution approach that utilizes geometric characteristics of signatures outperforms the chain code histogram and invariant moments both in retrieval score and the speed of building corresponding feature vectors. The search time of the proposed method and the chain code histogram method are near. Moment invariants show the fastest searching time but the recognition score is low. The times $t_{0}$ and $t_{1}$ turned out to be 842 and 7 milliseconds, respectively, using a Pentium-III, $1000 \mathrm{MHz}$ machine.

\section{CONCLUSION}

We introduced a new fast method for line segment extraction from images and utilized the method for Persian signature recognition. The proposed Chain Based Line Segment Extraction (CBLSE) method is utilizing a chain code representation of edge points and splits the chains in higher curvature points. Multiple chains are extracted from a thinned sketch 


\section{TENCON $2003 / 14$}
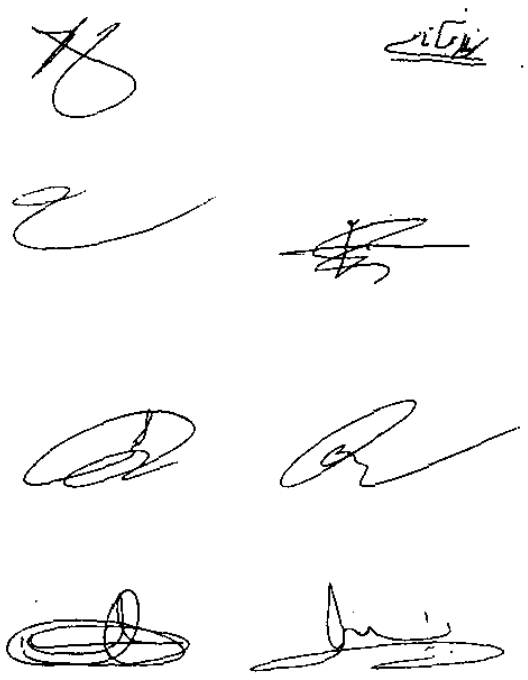
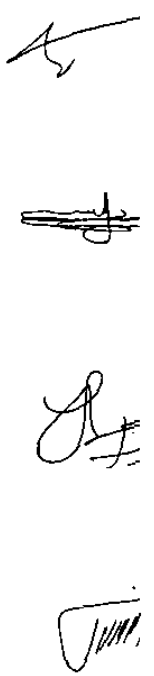

Figure 2. Cursive signature examples

Table 1. Comparison of Line Segment Distribution, Chain Code Histogram and Moment Invariants methods

\begin{tabular}{|c|c|c|c|}
\hline Method & $\begin{array}{c}\text { Recognition } \\
\text { Score\% }\end{array}$ & $\begin{array}{c}\text { Buiding } \\
\text { Time }\end{array}$ & $\begin{array}{c}\text { Searching } \\
\text { Time }\end{array}$ \\
\hline . Line Seg. Dis. & 97.35 & $t_{0}$ & $3.6 t_{1}$ \\
\hline Chain Code His. & 95.01 & $1.2 t_{0}$ & $3 t_{1}$ \\
\hline Moment Inv. & 79.31 & $6.7 t_{0}$ & $t_{1}$ \\
\hline \hline
\end{tabular}

(or from an edge map) of the input image. Each chain is then divided into several micro chains by a sequence of shifting, smoothing and differentiating stages. since each chain is processed individually, the method is suitable for use on parallel machines. The length and position distribution of the straight line segments connecting the end points of micro chains are used for recognition. Persian cursive signature recognition/retrieval was chosen as an application. The recognition score for the proposed method is better than for chain code histograms and invariant moments. The speed of the method is also reasonable. The approach can be embedded in paperless office projects for verification of Iranian signatures.

Banking systems can use the proposed approach for automatic validation of signed documents. The approach can be tailored for sketch-based image retrieval. In the latter application, the user provides a sketched images and looks for similar images in an image database.

\section{ACKNOWLEDGEMENTS}

The authors thank all participants who gave permission to use their signatures in this study. The first author is financially supported by the Ministry of Science, Research and Technology of I.R. Iran.

\section{REFERENCES}

[1] Y. Gao and M. K. H. Leung, "Face recognition using line edge map," IEEE Trans. Patt. Anal. and Mach. Intell, vol. 24, no. 6, pp. 764-779, 2002.

[2] A. Etemadi, "Robust segmentation of edge data," in IEE Int. Conf. Image Processing and its Applications., 1992, pp. 311-314.

[3] J.-S. Jang and K.-S. Hong, "Fast line segment grouping method for finding globally more favorable line seg. ments," Patt. Recog., vol. 35, no. 10, pp. 2235--2247, 2002.

[4] J. Zhu and Y. Zhao, "Vehicle license image segmentation using wavelet transform," in Proc. IEEE Int. Symposium on Intelligent Multimedia, Video and Speech Processing., 2001, pp. 267-70.

[5] J. B. Burns, A. R. Hanson, and E. M. Riseman, "Extracting straight lines," IEEE Trans. Patt. Anal. and Mach. Intell., vol. 18, no. 4, pp. 425-455, 1986.

[6] R. C. Nelson, "Finding line segments by stick growing," IEEE Trans. Patt. Anal. and Mach. Intell., vol. 16, no. 5, pp. 519-523, 1994. 


\section{Image Processing / 15}

[7] J. Assfalg, M. Bertini, C. Colombo, and A. D. Bimbo, "Semantic annotation of sports videos," IEEE Multimedia, vol. 9, no. 2, pp. 52-60, 2002.

[8] A. Mowlaei, K. Faez, and A.T. Haghighat, "Feature extraction with wavelet transform for recognition of isolated handwritten farsi/arabic characters and numerals," in proc. IEEE DSP, 2002, vol. 2, pp. 923-926.

[9] M.B. Menhaj and M. Adab, "Simultaneous segmentation and recognition of farsi/latin printed texts with mip," in proc. IEEE Int. Conf. Neural Networks, 2002, vol. 2, pp. 1534-1539.

[10] M. Dehghan, K. Faez, M. Ahmadi, and M. Shridhar, "Off-line unconstrained farsi handwritten word recognition using fuzzy vector quantization and hidden markov word models," in proc. IEEE 15th Int. Conf. Patt. Recog., 2000, vol. 2, pp. 351-354.

[11] A. Dehghani, F. Shabini, and P. Nava, "Off-line recognition of isolated persian handwritten characters using multiple hidden markov models," in proc. IEEE Int. Conf. Inform. Technology: Coding and Computing, 2001, pp. 506-510.

[12] M. R. Hashemi, O. Fatemi, and R. Safavi, "Persian cursive script recognition," in proc. IEEE Third Int. Conf. Document Anal. and Recog., 1995, vol. 2, pp. 869-873.

[13] A. D. Bimbo, Visual Inform. retrieval, Morgan Kaufmann Publishers, 1999.

[14] M. Dehghan and K. Faez, "Farsi handwritten character recognition with moment invariants," in proc. IEEE $D S P, 1997$, vol. 2, pp. 507-510.

[15] J. Iivarinen and A. Visa, "Shape recognition of irregular objects," in Proc. of Spie, 1996, vol. 2904, pp. 25-32.

[16] A. V. Da Silva and D. S. De Freitas, "Wavelet-based compared to function-based on-line signature verification," in proc.'IEEE Int. conf. Comput. Graphics and Image Processing, 2002, pp. 218-225.

[17] E. J. R. Justino, F. Bortolozzi, and R. Sabourin, "The interpersonal and intrapersonal variability influences on off- line signature verification using hmm," in proc. IEEE Int. conf. Comput. Graphics and Image Processing, 2002, pp. 197-202.

[18] J. Canny, "A computational approach to edge detection," IEEE Trans. Patt. Anal. and Mach. Intell., vol. PAMJ-8, no. 6, pp. 679-698, Nov. 1986.

[19] R. C. Gonzalez and R. E. Woods, Digital Image Processing, Addison-Wesley, 1992.

[20] H. Li, B. S. Manjunath, and S. K. Mitra, "A contour-based approach to multisensor image registration," IEEE Trans. Image Processing, vol. 4, no. 3, pp. 320-334, 1995.

[21] C.-H. Teh and R. T. Chin, "On image anal.ysis by the method of moments," IEEE Trans. Patt. Anal. and Mach. Intell., vol. 10, no. 4, pp. 496-513, 1988.
[22] A. Chalechale and A. Mertins, "An abstract image representation based on edge pixel neighborhood information (EPNI)," in Lecture Notes in Comput. Science. EurAsian-ICT, 2002, vol. 2510, pp. 67-74. 